\title{
Numerical pre-clinical testing in hard tissue surgery
}

\author{
J.Vander Sloten, H. Van Oosterwyck, V. Pattijn \& \\ T. Van Cleynenbreugel \\ Division of Biomechanics and Engineering Design. Katholieke \\ Universiteit Leuven, Celestijnenlaan 200A -3001 Heverlee (Belgium)
}

\section{Bone as a smart material}

Bone is more than just an engineering material. Bones provide stability to our body and are also an essential element in our mobility. Moreover bones act as reservoirs of important minerals such as calcium. From the macroscopic point of view there are two major types of bone tissue: the compact or cortical bone and the trabecular or spongy bone. The shaft or diaphysis of long bones is mostly made up of cortical bone, trabecular bone is found in the ends of bones (the epiphyseal region) and inside short bones.

From the engineering point of view, cortical bone consists of fibers (osteons) running in the length direction of the bone. This gives rise to mechanical properties reflecting this transverse isotropic structure. Typically cortical bone has an elastic modulus of $18 \mathrm{GPa}$ in the longitudinal direction and $11 \mathrm{GPa}$ in the two transverse directions i.e. radial and tangential [1].

The trabecular bone is a porous, honeycomb like structure. It has been observed already a long time ago [2], [3] that the directions of the struts that build this trabecular structure are not random, but show clear preferential orientations in each location within the bone. The so-called Wolff's Law states that the preferential orientations of trabecular bone coincide with the principal stress directions in the loaded bone structure at each place. As a result of this law or principle, the trabecular bone structure is such that it yields a maximum mechanical strength with a minimum of bone material [4]. The elastic modulus of trabecular bone is highly density dependent and may vary between wide ranges, e.g. between $100 \mathrm{MPa}$ and $1500 \mathrm{MPa}$ [5].

Bone is more than just a cleverly built material: it is able to adapt its structure and hence its properties to changes in the mechanical loads acting upon it. This is known as adaptive bone remodelling [6]. There seems to exist some kind of optimal loading condition on the bones (magnitude and direction of stresses, frequency content) for which there is no net remodelling activity. The normal 
bone turnover, consisting of removal of small amounts of bone and subsequent repair by the generation of new bone, assures that the overall geometry and inner structure of bones are maintained. If however the loading deviates from the optimal loading, a net remodelling activity is observed. If bone is loaded higher than it was used to, higher stresses than normal are engendered in the bone and extra amounts of new bone will be laid down, resulting in an increase of bone mass and volume. Reversely, underloading of bone will yield a net bone resorption. A number of animal experiments that studied the bone adaptative response around either oral or transcutaneous implants, have shown that overload can lead to excessive marginal bone resorption or even complete loss of osseointegration [7], [8].

\section{The mechanosensing mechanism}

Obviously, bone must be able to sense the mechanical loading acting upon it. This is called the mechanosensing capacity of bones. The most recent theories point towards the existence of a population of cells within the bone, that act as a sensing network for the local stresses and strains. Most probably, the osteocytes constitute the mechanosensing network [9], [10]. Through the canaliculi, the lacunae in which the osteocytes reside are connected to each other. Moreover, this network is also able to trigger the response of the osteoblast and osteoclast cells that cover the free bone surfaces (e.g. the periosteal and endosteal cortical bone surface). These cells are the sources of new bone deposition (osteoblasts) and removal of bone (osteoclasts). Within this network of lacunae and canaliculi, extracellular fluid flow is induced by dynamic mechanical loading. This fluid flow, combined with the electro-kinetic effects induced by it (under the form of streaming potentials) may well be the mechanism behind the mechanosensing capacity of the bone [11]. Also Cowin and coworkers [12] point at the stimulation of osteocytes by the relatively small fluid shear stresses acting on the membranes of their osteocytic processes. Since the fluid shear stress is nearly proportional to the product of frequency and strain, they expect a similar response at 0.1 percent strain and $2 \mathrm{~Hz}$ loading, and at 0.01 percent strain and 20 $\mathrm{Hz}$ loading on the bone. Bone indeed seems to be particularly sensitive to loading at low strains and relatively high frequencies as demonstrated e.g. by Rubin et al. in 2001 [13].

The processes involved in the adaptive bone remodelling also play a role in the repair of fractured bones (regeneration capacity) and in the osteogenesis in embryonal development. In fracture healing, the dead bone is first removed, and then a cartilaginous bridge is established between the fractured parts by the action of chondrocytes. Because of the combined action of osteoclasts, osteoblasts, chondrocytes and mineralisation processes, new bone is formed that will replace the cartilaginous bridge by a bony connection, causing the fracture to heal. This process of regeneration is controlled by the mechanical environment that is created in the tissue in the fractured zone. For this reason, a fracture is stabilized by a plaster or by internal or external fixation during the healing process. Several authors have studied the influence of mechanical loading (e.g. the amounts of relative motion allowed in the fracture healing area, the straining 
of the tissue in the fracture area) upon the healing process [14]. Also in embryology, there is growing evidence that the mechanical loadings imposed upon the foetal developing bones (here too the bone tissue starts from a cartilaginous core) influence the differentiation of the cartilaginous tissue towards bone tissue [15]. The loadings upon the foetal bones are then caused by muscle contractions in utero. The research into the effects of mechanical loading upon the tissue differentiation process is very important, and of fundamental importance for the whole area of tissue engineering for the generation of bone and cartilage.

\section{Numeric pre-clinical testing and tissue differentiation}

Engineers like to translate concepts into formulas since it allows to quantify concepts that otherwise would look rather abstract. Cowin [16] was amongst the first to propose formulas to describe the adaptive bone remodelling e.g. $\mathrm{U}(\mathrm{Q})=$ $\operatorname{Cij}(Q)\left[e_{i j}(Q)-e_{i j}{ }^{o}(Q)\right]$ meaning that the velocity of the movement of a point $Q$ on the bone surface is proportional to the difference between the actual strain value acting at a certain point on the bone and a reference value for this strain. The range of stresses, strains or mechanical stimuli in general for which bone shows no tendency for adaptive bone remodelling is called the lazy region or the quiet region. Other researchers have tried to model the adaptive bone remodelling process with a continuum damage and fatigue model [17].

The availability of reliable models to describe adaptive bone remodelling gives rise to numerical algorithms that simulate the events taking place at the bone surface and within the bone as a function of time. Iterative computer algorithms can be designed that feed back stresses and strains calculated with the help of e.g. finite element models of bone structures or bone-implant structures, to changes in bone geometry or bone structure (Figure 1).

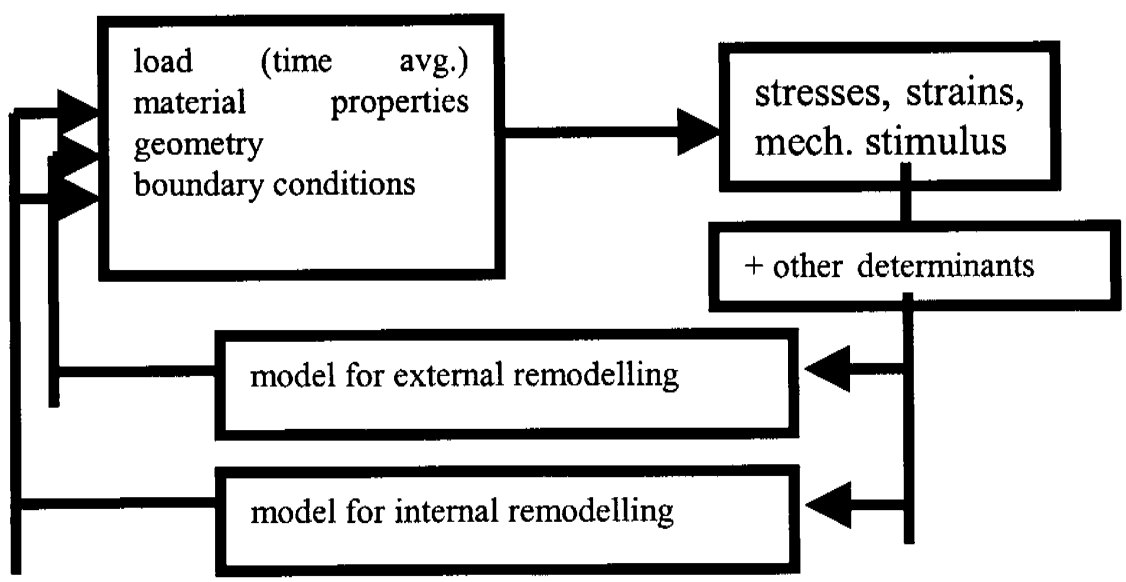

Figure 1: General layout of an iterative algorithm to simulate adaptive bone remodelling with the capacity to simulate both internal bone remodelling and surface bone remodelling. 


\section{8}

Simulations in Biomedicine $V$

Each loop through this algorithm then simulates one time step. In earlier years, the numerical models to describe the adaptive bone remodelling were rather phenomenological. They described relations between an input (a measure for local bone load) and an output (a change in bone geometry or material property) without having a clear relationship with the biological principles that underlie these remodelling processes. Recently, the understanding of the biology of tissue differentiation, and the role of mechanical loading and mechanosensing in this tissue differentiation process is gaining importance (see also Huiskes, 2000 [18]).

\section{Case studies}

\subsection{Adaptive bone remodelling around screw shaped dental implants.}

Inappropriate implant loading has been suggested as one of the most important factors for late failures of endosseous oral implants. Animal experimental research has demonstrated that overload can lead to excessive marginal bone loss or even complete loss of implant osseointegration [7]. Some clinical findings seem to confirm the overload theory [19] although others have suggested underload as a possible mechanism for marginal bone loss [20]. Our group has investigated the bone stress and strain distribution around screw shaped implants during functional loading by means of the finite element method [21].

Results were available from an animal experiment to study the bone reponse to cyclic loading using a rabbit tibia model and a screw shaped implant. Highresolution finite element models, based on microfocus computed tomography $(\mu \mathrm{CT})$ of post-mortem samples, were created to incorporate the exact bone structure around the implants. Alternatively, procedures were developed to build patient-dependent, anatomical models of an entire jaw, restored with implantsupported prostheses. Jaw anatomy and implant position were derived from medical computed tomography (CT) images and were incorporated in the models by applying computer-aided design (CAD) techniques. Heterogeneous bone elastic properties were implemented by means of a relationship between the CT numbers and the Young's modulus. For each individual patient implant loads during function were measured by means of instrumented abutments and were applied in the patient-dependent finite element model. In this way, in vivo bone loading patterns were calculated for an individual patient.

It was found that in case of titanium implants with a smooth surface, the implantbone interfacial tensile strength is exceeded at the implant neck, even for physiological load levels. As a consequence, interfacial debonding and relative motion (micromotion) occur at the marginal bone level. Stress and strain results suggested that underload (disuse atrophy) is not important for the explanation of marginal bone loss. Furthermore, the risk of marginal bone overload (fatigue) seems to be low in case of physiological loading conditions. These results were consistent with the results from an earlier study on bone stresses around solitary screw shaped oral implants. However, another finite element study, in which the 


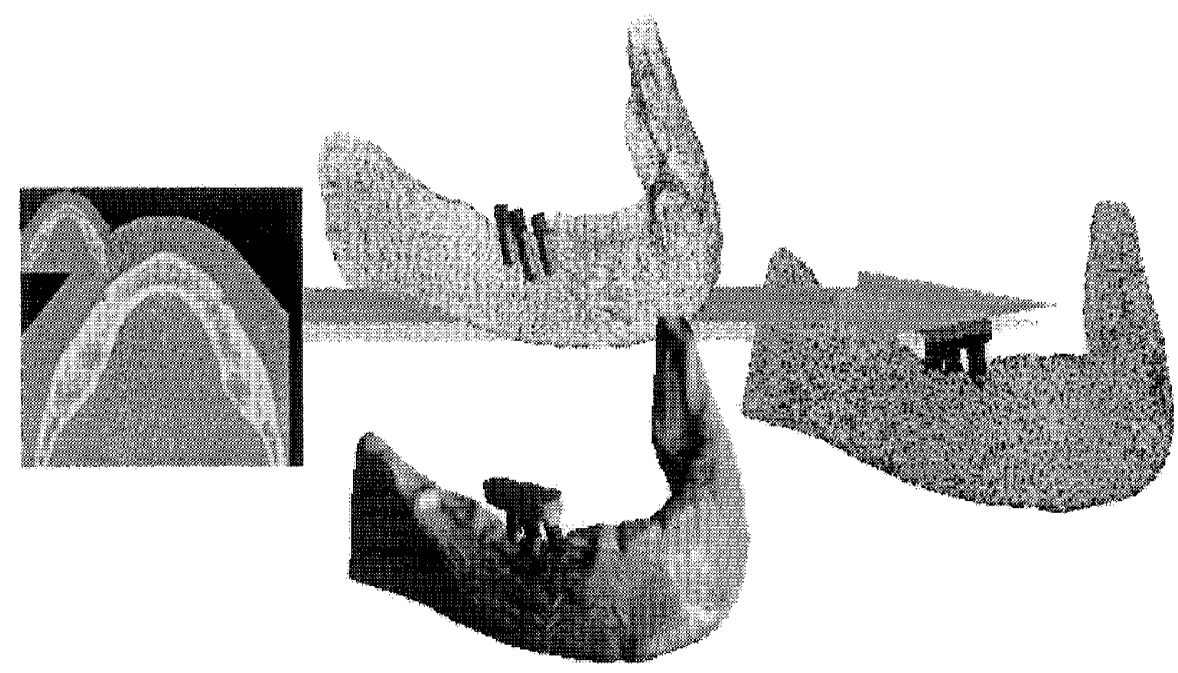

Figure 2: Medical image based generation of finite element models of a mandible with oral implants and fitted with a prosthesis.

bone stress and strain distribution was studied around implants, placed in a compromised jaw anatomy (occurrence of dehiscences) revealed that for such patients a strong increase in stress and strain can be expected at the site of the dehiscence. Although the $\mu \mathrm{CT}$-based finite element model of the animal experiment demonstrated that the occurrence of high bone stresses and strains are related to bone resorption it is yet unclear whether such high bone loads can be encountered clinically. Therefore, it is hypothesised that interface debonding and subsequent relative motion contribute to marginal bone loss.

\subsection{Hip osteotomy planning}

Skeletal malformations of the pelvis and/or the proximal femur in young children could lead to dislocation of the hip, further deformation of the hip joint, arthrosis, etc. To avoid these problems and allow a normal biomechanical function of the hip joint a surgical intervention is necessary.

For an optimal post-operative result the surgeon has to take into account the biomechanics of the hip joint during the pre-operative planning. Two main parameters characterise the hip joint biomechanically: (1) the joint reaction force on the femur head and (2) the contact area between femur head and acetabulum. Both determine the stress distribution in the proximal femur.

These two parameters are pre-operatively evaluated to enhance the choice of the most suitable surgical treatment.

Based on the CT images of the hip joint a three dimensional model of pelvis and femur is made. Combining this patient-dependent model with a mathematical model of the muscles allows the calculation of the femoral joint force for a specific loading condition e.g. standing on one leg. Thus both the magnitude and 
the direction of the joint force are determined. Comparison with reference values, i.e. values for a normal hip joint of a healthy person, indicates the necessity of a femoral osteotomy. After simulation of the osteotomy on the three dimensional model, the femoral joint force is recalculated and compared with the preoperative value as well as the reference value. This process is repeated until the postulated surgical treatment optimizes the reaction force on the femur head towards magnitude and direction. It is clear that the surgical intervention must still be within the limits of the orthopaedic feasibility.

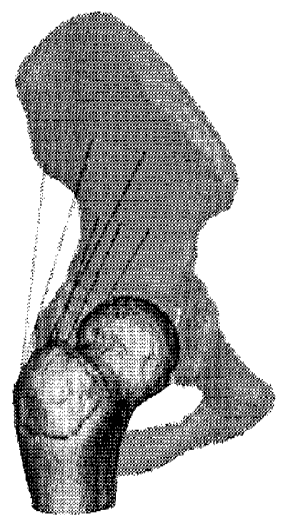

Figure 3: 3D model of pelvis and femur. Muscle directions are also indicated.

Furthermore the three dimensional patient-dependent model allows the determination of the contact area, which can also be compared with a reference value. If necessary the contact surface can be enlarged by a shelf arthroplasty or a pelvis osteotomy. The latter can also be pre-operatively simulated and evaluated.

\subsection{Biomechanics of scaffold design for bone tissue engineering}

Microfocus Computed Tomography $(\mu \mathrm{CT})$ images of a spongy bone sample can be used to construct a high-resolution numerical model of trabecular bone. This model contains the geometrical information of the tissue at the individual trabeculae. Relying on the mechanical properties of trabecular bone tissue, this model can serve as a tool for the investigation of the overall mechanical properties of a bone sample and its behaviour under various mechanical loading conditions. This is done using finite element analysis.

Large defects in the bones of the human body, for example after tumour resection, weaken the bone and must be repaired. A new technique makes use of a so-called 'bone scaffold', an artificial structure with which the defect is filled. After a certain period the scaffold is broken down and replaced by newly formed bone and the defect is healed. Before complete healing, the scaffold takes over the load bearing function of the bone tissue in the cavity. However, this implies that the scaffold must have mechanical properties that are comparable to those of spongy bone. A too low stiffness will deprive the bone from mechanical support; a scaffold that is too stiff will cause 'stress shielding': the scaffold carries the 
entire load, thereby diminishing the load in the surrounding healthy bone tissue. As mentioned previously, this will cause a resorption of the healthy bone. Also the lower deformation of a stiff scaffold will cause less activation of the osteoblasts that reside on the scaffold and hence the apposition of new bone on the scaffold will be slower than desired.

The solution to this problem lies in the comparison of the mechanical properties of the scaffold with the properties obtained from the numerical analysis of healthy bone (Figure 4). This comparison allows the design of a scaffold that has mechanical properties that match those of spongy bone. This ensures an optimal design of the scaffold according to its function: the temporary replacement of bone tissue in load bearing bones [22].

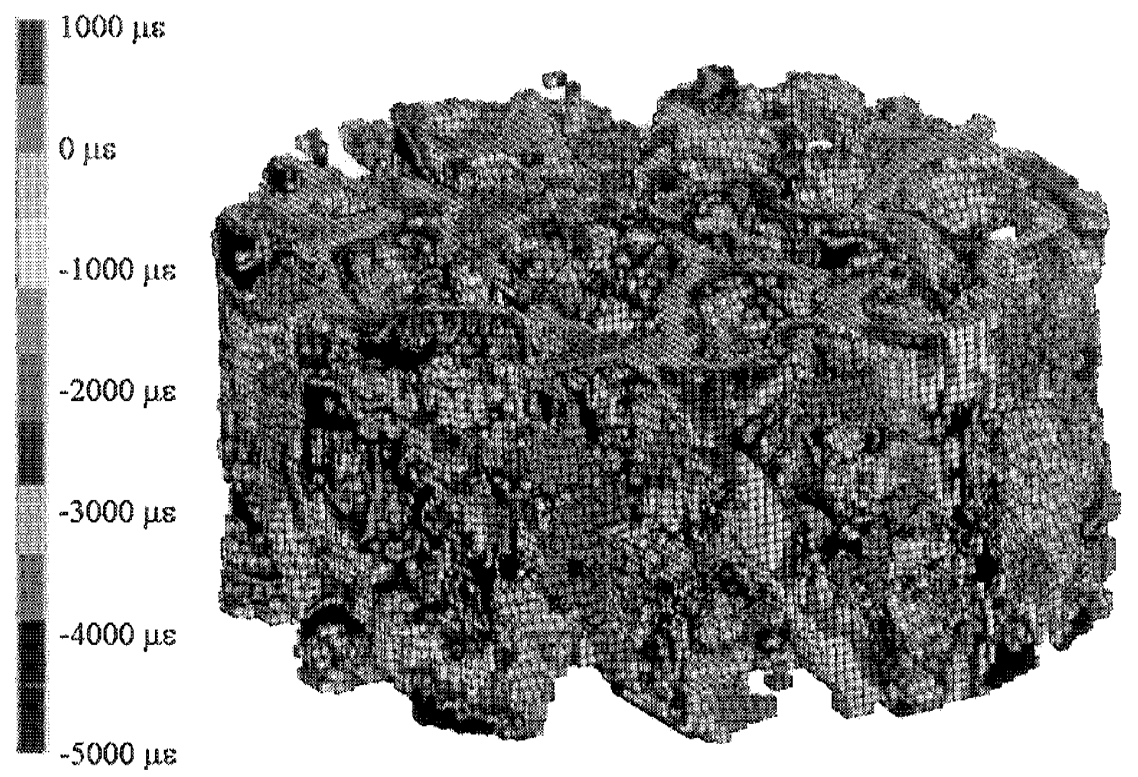

Figure 4: Vertical strain in trabecular bone sample subjected to 4000 microstrain, macroscopic compression (sample height $2.7 \mathrm{~mm}$ ).

\section{Conclusion}

The musculoskeletal system is an important constituent of our body. Besides of providing an optimal supporting structure for stability and movement, it is also able to maintain this optimality when the mechanical conditions change. Through the process of adaptive bone remodelling, bone geometry and structure may be regulated to keep a local mechanical stimulus in an optimal range. Numerical models have been proposed that describe the adaptive bone remodelling and relate it to various types of mechanical stimulus. Recently however, attempts have been made to integrate the biology behind the adaptive bone remodelling into these models. This has given rise to models that are able to simulate tissue differentiation: they express changes in material composition 
(fluid, fibrous and mineral phases) and relate them to parameters such as local damage, fluid flow, tissue deformation. Only when the numerical models help us to truly understand the complex events of tissue modelling, remodelling and differentiation, they will become useful tools in the development of new treatments, implants and other medical devices. Experimental validation by animal studies, studies using bone chambers and controlled patient studies are essential to make progress in the area of numeric simulation of adaptive tissue remodelling processes. Numeric pre-clinical testing will become reality only with the availability of reliable and quantitative models that incorporate the complex behaviour of our biological system. As such it will contribute to developing new medical procedures faster and cheaper, for the benefit of patient care and health care in general.

\section{References}

[1] Reilly DT and Burstein AH, The elastic and ultimate properties of compact bone tissue, Journal of Biomechanics 8, 393-405, 1975.

[2] Meyer GH, Die Architectur der Spongiosa, Archiv für pathologische Anatomie, Physiologie und wissenschaftliche Medizin (Reichert und Dubois Reymonds Archiv) 34, 615-628, 1867.

[3] Wolff J, Zur Knochenwachstumfrage, Archiv für pathologische Anatomie und Physiologie und für klinische Medizin (Virchov's Archiv) 50, 417-456, 1874.

[4] Vander Sloten J and Van der Perre G, Trabecular structure compared to stress trajectories in the proximal femur and the calcaneus, Journal of Biomedical Engineering 11, 203-206, 1989.

[5] Martens M, Van Audekercke R, Delport P, De Meester P, Mulier J, The mechanical characteristics of cancellous bone at the upper femoral region, Journal of Biomechanics 16, 971-983, 1983.

[6] Lanyon L., Functional strain in bone tissue, Journal of Biomechanics 20, 1083-1093, 1987.

[7] Isidor F., Loss of osseointegration caused by occlusal load of oral implants: a clinical and radiographic study in monkeys, Clinical Oral Implants Research 7, 143-152, 1996.

[8] Duyck J., Ronold, H., Van Oosterwyck, H., Naert, I., Vander Sloten, Ellingsen, J.E. The influence of static and dynamic loading on the marginal bone behaviour around osseointegrated implants: an animal experimental study, Clinical Oral Implant Research, Vol. 12 No. 3, pp. 207-218, 2001.

[9] Cowin S. Candidates for the mechanosensory system in bone, Journal of Biomechanical Engineering 113, 191-197, 1991.

[10] Burger EH, Klein-Nulend J., Mechanotransduction in bones - role of the lacuno-canalicular network, FASEB Journal 13, S101-112, 1999.

[11] Knothe-Tate ML, Steck R, Forwood MR, Niederer P. In vivo demonstration of load-induced fluid flow in the rat tibia and its potential implications for processes associated with functional adaptation, Journal of Experimental Biology 203, 2737-2745, 2000. 
[12] Cowin S., Bones have ears, Acta of Bioengineering and Biomechanics, Proc. $13^{\text {th }}$ Conference of the European Society of Biomechanics, pp. 29-31, 2002.

[13] Rubin CT, Turner S, Bain S, Mallinckrodt C, McLeod K. Low mechanical signals strengthen long bones, Nature, 412, 603-604, 2001.

[14] Claes LE and Heigele CA. Magnitudes of local stress and strain along bone surfaces predict the course and type of fracture healing, Journal of Biomechanics 32, 255-266, 1999.

[15] Carter D and Beaupré G. Skeletal function and form: mechanobiology of skeletal development, aging and regeneration, Cambridge University Press, 2000.

[16] Cowin S and Van Buskirk WC. Surface bone remodelling induced by a medullary pin, Journal of Biomechanics 12, 269-276, 1979.

[17] Prendergast, P., Taylor, D. Prediction of bone adaptation using damage accumulation, $J$. of Biomechanics 27: 1067-1076, 1994.

[18] Huiskes R. Challenges in bone biomechanics, inaugural lecture Technical University of Eindhoven, The Netherlands, 2000.

[19] Quirynen M, Naert I, van Steenberghe D. Fixture design and overload influence marginal bone loss and fixture success in the Brånemark system, Clinical Oral Implants Research 3, 104-111, 1992.

[20] Vaillancourt H, Pilliar RM, McCammond D Factors affecting crestal bone loss with dental implants partially covered with a porous coating: a finite element analysis, International Journal of Oral and Maxillofacial Implants 11, 351-359, 1996.

[21] Van Oosterwyck H, Duyck J, Vander Sloten J, De Cooman M, Lievens S, Puers R, Van der Perre G, Naert I. The influence of bone mechanical properties and implant fixation upon bone loading around oral implants, Clinical Oral Implant Research 9, 407-418, 1998.

[22] Van Cleynenbreugel T, Vander Sloten J., Van der Perre G, Luyten F. Trabecular bone scaffolding using a biomimetic approach, Journal of Materials Science: Materials in Medicine 13, 1245-1249, 2002. 
